\title{
Un colloque en sciences du langage en ligne : éléments d'évaluation
}

\author{
Benoît Habert \\ ILF - ENS LSH \& ICAR \\ benoit.habert@ens-1sh.fr \\ Valelia Muni Toke \\ MoDyCo \& Université Paris 10 \\ valelia.muni toke@u-paris10.fr \\ Nicolas Grekas \\ Intellagence \\ nicolas.grekas@intellagence.eu
}

\section{Introduction}

L'ILF a souhaité pour le Congrès mondial de linguistique française avoir recours à une plateforme logicielle permettant de gérer explicitement la sélection des communications, la fabrication matérielle des actes et les facilitant : réception en ligne des propositions de communication, organisation du circuit de relecture, mise en forme présentationnelle des contributions acceptées et des contributions complémentaires (introductions aux tables rondes, conférences invitées), préparation des fichiers nécessaires, production du CD-ROM des actes et du site correspondant, etc.

L'ILF a choisi la solution commerciale Accélérateur de congrès (http://congres.intellagence.eu/) de la société Intellagence (http://congres.intellagence.eu/apropos). Le choix d'une solution commerciale était motivé par le souhait de disposer effectivement d'une assistance à l'utilisation de ce type d'environnement pendant le congrès.

Le projet de la société Intellagence est d'offrir à la communauté scientifique et universitaire des outils en ligne facilitant les échanges entre chercheurs dans le cadre de l'élaboration de congrès, de programmes de recherche, et dans celui de la gestion de sociétés savantes, etc. Selon Intellagence, de nombreuses sociétés Internet développent des innovations dans le domaine de la recherche documentaire, mais très peu dans le domaine des outils de gestion, voire de «production» scientifique. Or, l'évolution de la vie des chercheurs les conduit à devoir de plus en plus consacrer de temps à la gestion de projets ou d'événements, de groupes de travail ainsi qu'à la valorisation et à la diffusion de leurs travaux, dont le succès favorise les évaluations et par là les financements.

Le produit Accélérateur de Congrès se veut modulaire et adaptable. En ce sens, Intellagence entend être un prestataire de type $S a a S$, acronyme anglais de «Software as a Service ». Ses équipes ont été amenées à faire évoluer le produit standard pour l'adapter aux choix stratégiques et scientifiques du CMLF. La notion de service tient également à la pédagogie du produit, qui doit être faite auprès des personnes en charge du congrès, mais aussi souvent auprès des membres de la communauté scientifique utilisatrice. Cette expérience dans le monde des linguistes est assez similaire à celles vécues dans d'autres communautés scientifiques : une partie importante de la population a des difficultés au premier abord avec l'outil. En revanche, pour Intellagence, la fidélité de l'ensemble de ses clients confirme qu'Accélérateur de congrès offre une véritable valeur ajoutée à l'événement, en pérennisant les liens entre les personnes, en augmentant, quantitativement et qualitativement les productions et leur diffusion.

Le présent article fait le bilan de l'appel à une telle plateforme pour les objectifs visés : 
- une procédure d'évaluation objectivable, c'est-à-dire qui permette de rendre compte avec le maximum de détail possible des choix effectués ;

- des actes disponibles à l'ouverture du congrès sur CD-ROM inséré dans le livret des résumés ;

- un site pérenne.

\section{Le dépôt des propositions de communication}

\subsection{Les propositions reçues : repères}

Le nombre total de propositions reçu, 261, a été inférieur à ce qui avait été souhaité. Plusieurs facteurs ont dû jouer. Certains relèvent du positionnement global du congrès, d'autres des modalités de communication demandées.

Les colloques « généralistes » ne sont pas légion, en particulier en linguistique française. Il n'est donc pas aisé, malgré un découpage en thématiques et un relais vers des circuits de communication plus spécialisés, d'attirer vers un tel type de rencontre, a fortiori quand il s'agit de la première édition.

Certaines thématiques disposent en outre de rencontres récurrentes et de supports de publication plus naturels. S'il y a eu très peu de communications touchant au traitement automatique des langues, par exemple, c'est à la fois parce que l'appel à communications indiquait que le TAL n'était pas une thématique en tant que telle et parce que la communauté francophone du TAL dispose d'une conférence annuelle maintenant bien établie: TALN (voir par exemple pour l'édition 2007: http://www.irit.fr/taln07/), d'une association spécifique, l'ATALA (http://www.atala.org/) et de sa revue (en ligne désormais : http://www.atala.org/-Revue-TAL-). Des explications similaires valent peut-être pour les thématiques de la phonétique/phonologie et de la morphologie, qui ont donné lieu à peu de propositions.

Pour certaines thématiques, ce serait le cas de la phonétique/phonologie, les travaux ne se centrent pas forcément sur le français en tant que tel, ce qui rendait moins évident l'envoi de propositions au CMLF.

L'habitude n'est pas, dans plusieurs domaines des sciences du langage, à une sélection sur des propositions longues. L'appel à propositions est en outre arrivé trop tard. Une partie des personnes potentiellement intéressées n'ont pas eu matériellement le temps de rédiger une proposition complète. Il aurait probablement fallu 6 mois entre l'appel à communications et le dépôt des propositions. Le délai pour cette édition a été de la moitié : c'est trop court, surtout pour un mode de publication différant des « coutumes ». La longueur minimale des propositions était en effet de 10 pages, en police 10 points, interligne simple. Si certains auteurs ont visiblement vu dans ces modalités de proposition l'opportunité de soumettre des pages déjà en cours d'écriture avant le congrès, voire des propositions ayant déjà fait l'objet de communications dans d'autres colloques, la majorité des auteurs a vu dans la longueur du papier demandé une contrainte parfois hors de portée, du fait des échéances imposées. Certes, la date limite de dépôt, initialement prévue le 4 janvier, a été repoussée d'une dizaine de jours, et le serveur laissait une marge (il ne fermait pas brutalement à minuit, contrairement aux applications en ligne destinées aux recrutements ou aux inscriptions à des concours). Une dizaine de propositions a en outre été déposée directement par le comité technique, après la fermeture du système. En revanche, une dizaine d'auteurs a laissé sur le site de dépôt une proposition de moins de cinq pages, sans trouver le temps de la compléter.

La demande d'anonymat des propositions a également déconcerté. Elle n'est pas non plus « traditionnelle », puisque l'anonymat demandé aux auteurs était «total»: il s'agissait de retirer du papier toute mention (affiliation, nom des corpus, référence bibliographique) qui comportât leur nom. Sa définition initiale sur le site de l'ILF n'était pas suffisamment précise. Les modalités de son application ont donc donné lieu à discussion avec les auteurs : faut-il aussi supprimer les auto-citations et autoréférences bibliographiques (ce manque, dans une bibliographie, de l'auteur dont on sait qu'il est 
spécialiste, le signalerait d'emblée comme auteur du papier) alors qu'à l'inverse, ne pas citer une publication connue du domaine et dont on se trouve être l'auteur peut donner l'impression au relecteur du texte dûment anonymisé qu'on ne maitrise pas bien la bibliographie du domaine ? Cependant, ce dernier cas de figure n'a pas, sauf erreur, été rencontré lors des relectures. De même, l'idée selon laquelle la communauté sait qui travaille sur quoi, et selon laquelle le thème de recherche du papier constituerait également un indice fiable quant à l'identité de l'auteur n'a pas été vérifiée : après discussion avec les comités thématiques et de relecture, il s'avère que, bien souvent, l'identité de l'auteur, révélée à l'issue du processus de sélection, a constitué une surprise. On peut se demander par ailleurs si l'anonymat n'a pas découragé des (enseignants-)chercheurs « reconnus » de proposer une communication et de risquer d'être rejetés. Ainsi, les modalités de l'anonymat restent à comparer avec les pratiques d'autres champs disciplinaires, dans lesquels il est toujours la règle. Il reste que ce choix du CMLF a été bien reçu de la part des jeunes chercheurs, mais beaucoup moins de la part des chercheurs confirmés. Il y aurait là matière à une plus ample discussion, qui permettrait de comprendre quels sont les arguments de part et d'autre, et quels sont les avantages et inconvénients respectifs pour les individus concernés.

Le nombre plus restreint qu'escompté de propositions a poussé le comité de programme à assortir chaque communication acceptée d'une présentation orale, alors qu'au départ l'idée était que chaque communication acceptée donne lieu à un article complet dans les actes, mais qu'une partie seulement soit présentée oralement. Pour les thématiques où le nombre de propositions a été finalement réduit, la table ronde de conclusion a changé de statut: il s'agit moins de tirer les enseignements des communications que de fournir un état de la thématique ou des points de vue complémentaires.

\subsection{Les modalités du dépôt en ligne des propositions}

Les auteurs devaient s'inscrire via le site de dépôt : le serveur leur fournissait automatiquement un login et un mot de passe, lesquels permettaient ensuite de redéposer autant de fois que nécessaire le texte, et donc de le modifier jusqu'à la date limite, fixée au 14 janvier. Les textes devaient en outre suivre la feuille de style mise en ligne sur le site du congrès.

Les renseignements demandés lors de l'inscription incluaient le résumé de la proposition (400 mots), les mots-clés et les besoins en matériel pour la communication orale proprement dite.

Les chercheurs français devaient indiquer leur laboratoire d'affiliation dans la base de données de référence, conforme à celle de HAL, l'Hyper-Archive en Ligne du CNRS (http://hal.archivesouvertes.fr/). Les chercheurs étrangers entraient manuellement l'adresse de leur établissement de rattachement.

\subsection{Les problèmes rencontrés}

S'il s'agit ici de mettre en évidence les problème rencontrés, de telle sorte que les éditions suivantes puissent les prévoir et les contourner, il est tout de même à signaler que, sur 261 propositions déposées, la moitié d'entre elles n'a pas posé de difficulté. L'inscription elle-même (avant le dépôt du texte) a posé problème à une quinzaine de personnes, pour lesquelles elle a été faite directement par le comité technique ; en cause, l'incompatibilité de certains navigateurs avec la plateforme, ou bien le dépassement de la date limite.

\subsubsection{L'affiliation}

La base de données utilisées par la plateforme imposait que les chercheurs s'identifient par leur appartenance à un laboratoire : or, si toutes les UMR sont dûment enregistrées dans HAL, il s'est avéré que quelques EA (équipes d'accueil) ou JE (Jeunes Equipes) n'étaient pas répertoriées. De plus, les enseignants-chercheurs montrent dans l'ensemble un attachement plus fort à leur lieu d'exercice de l'enseignement, ie. l'université, et se sont parfois trouvés déconcertés par cette exigence de rattachement à une unité de recherche. On observera donc, dans les actes, un décalage entre l'affiliation minimale telle 
qu'elle figure dans le livret des résumés, et celle que les auteurs ont rédigée librement au début de leur article.

Ce problème n'est pas anodin car il touche, outre à la question de l'attachement (ou plutôt la conscience d'être attaché de façon privilégiée) des chercheurs à telle ou telle des institutions pour lesquelles ils travaillent, à celle, cruciale dans le contexte actuel d'évaluation et d'archivage des productions scientifiques, de l'indexation des articles. On sait ainsi qu'une des (multiples) raisons pour lesquelles les classements bibliométriques sont discutés est qu'ils se heurtent à l'extrême diversité des écritures possibles pour une même affiliation (jusqu'à une quarantaine), ce qui, malgré les normalisations a posteriori des institutions chargées des mesures, rend fragile un bon nombre de calculs concernant la production scientifique des chercheurs.

\subsubsection{Les difficultés techniques et le rapport à la « machine »}

Le choix d'une publication rapide et d'une mise à disposition des textes dès l'ouverture du congrès imposait que les textes soient, dès leur dépôt, mis en forme selon une feuille de style prédéfinie. Or, l'utilisation de celle-ci, mise à disposition sur le site du congrès, a mis en difficulté certains utilisateurs.

Certes, la feuille de style a été mise en ligne très (trop) rapidement, avec un champ pour le résumé et pour le nom des auteurs, ce qui contredisait d'une part les modalités de dépôt (les résumés étaient à entrer dans le formulaire d'inscription, pour faciliter l'édition du livret des résumés, distribué le jour du Congrès), et d'autre part l'exigence d'anonymat. La nouvelle feuille de style a ensuite été mise en ligne, ce qui a obligé une douzaine d'auteurs à reprendre leur texte sur les points précités. Néanmoins, ces difficultés ont pu être dépassées; les organisateurs puis les relecteurs ont en outre effectué un travail de correction en vue de la publication (une partie de la grille d'évaluation était consacrée à cet aspect présentationnel). La nécessité d'une homogénéité des actes n'a en fait pas semblé toujours très claire aux auteurs. Ainsi, une part importante des propositions, un cinquième environ, ne respectait pas la feuille de style ou l'anonymat: pour autant, le système ne les ayant pas repérées automatiquement, ces propositions ont souvent été déposées sans problème. Le comité technique a dû procéder à une relecture rapide de chaque document, afin de permettre la correction des problèmes les plus saillants, notamment l'anonymat, avant la relecture proprement dite.

Le système de dépôt était paramétré pour repérer automatiquement les erreurs de marge : la marge haute est en effet réservée à l'insertion, au moment de la publication, d'un en-tête comportant notamment le titre du colloque ; les marges droite et gauche doivent rester vides pour que la transformation en .pdf s'effectue correctement et que l'affichage final soit lisible; le pied de page ne doit pas contenir de numéros de page, puisque la numérotation est globale et calculée à partir des documents finaux publiés.

En conséquence, un certain nombre de textes se sont trouvés automatiquement rejetés par le serveur : si les premiers dépôts ont permis de signaler que le système était trop sensible (il a donc été paramétré à nouveau), les suivants ont mis en évidence des erreurs de mise en page : i) des tableaux, schémas ou images dépassaient dans les marges gauche ou droite ; ii) la feuille de style n'avait pas été respectée, et des styles comportant un retrait à gauche ou à droite étaient utilisés. Certes, le fait que Word permette un dépassement dans les marges alors que celles-ci ont été correctement paramétrées dans l'onglet « Mise en page » est une particularité dont les utilisateurs occasionnels n'ont pas forcément connaissance. L'instabilité de la feuille de style (c'est-à-dire le fait qu'un style pourtant prédéfini se trouve modifié par le simple passage du document d'un ordinateur à un autre) entre dans la même catégorie de « défauts » non prévisibles par l'utilisateur.

Tous ces problèmes ont pu être réglés : au total, sur 261dépôts, tous les textes ont été ouverts, vérifiés et éventuellement modifiés, et une quarantaine de textes (rejetés au départ par le serveur) a été corrigée par le comité technique puis redéposée sur le site.

La mise à disposition, sur le site du congrès, d'un guide d'utilisation de la feuille de style, n'a pas vraiment été ressentie comme utile, si l'on en juge par le nombre de messages reçus à l'adresse éléctronique réservée à l'appui aux contributeurs. De nombreux messages consistaient en effet en des 
questions dont les réponses figuraient explicitement dans le guide. Ce dernier est donc apparu comme un document visiblement trop long, et la majorité des auteurs ne l'ont pas lu. On pourra s'interroger sur ce fait : le site du congrès est sans doute peu lisible (mais sa mise en page est tributaire du paramétrage établi par le CNRS pour certains de ses sites). Cependant, la lecture de ce guide faisait partie des instructions explicites aux auteurs, et c'est donc l'existence même de ces instructions qui a posé problème.

Il semble ainsi qu'une part non négligeable des auteurs ait vu dans ces exigences techniques un symptôme de la «déshumanisation » de la recherche, de la gestion «par la machine » de problèmes scientifiques qui se trouveraient dès lors relégués au second plan. On peut tout à fait entendre ces inquiétudes. Il reste néanmoins que publier près de 3000 pages (en moyenne 15 pages pour chacune des 150 propositions retenues, plus les textes des tables rondes des douze thématiques, et les conférences invitées) en version papier n'est pas réalisable dans les délais qui ont été ceux du CMLF ; de même, recevoir par courrier plus de mille formulaires d'évaluation (si l'on compte que, pour chacune des propositions, il y a deux formulaires, un privé un public, remplis par chacun des relecteurs, deux en moyenne, jusqu'à quatre pour certaines propositions) ou traiter manuellement les informations nominatives, les besoins en matériel, les mots-clés et résumés attachés aux 261 propositions est impossible. Et il se trouve que, malgré l'aide précieuse de la plateforme informatique, la gestion de ces données reste lourde et n'est pas exempte d'erreurs diverses, l'humain restant finalement bien présent derrière cette automatisation.

Ainsi, la question du rapport à la «machine » s'est trouvé au cœur des difficultés dont les auteurs ont fait part dans leurs courriers à l'adresse électronique mise à leur disposition. Certains messages, peu amènes, imposent de réfléchir à la mise en place d'une interface plus conviviale : par exemple, les messages automatiquement générés par le serveur devraient être signalés plus clairement comme tels (pour que les auteurs n'y répondent pas comme s'ils avaient été pensés et adaptés en conséquence, par un humain). En particulier, les messages d'erreur (envoyés pour cause de dépassement dans les marges par exemple) sont à la fois extrêmement précis (ils signalent le numéro de la page, la position de la marge à corriger) et confus (ils ne donnent pas la marche à suivre pour la correction demandée).

\subsubsection{Le choix de Word, un problème idéologique ?}

Plusieurs auteurs, habitués à travailler avec des logiciels «libres» voire open source, ont remis en question le choix du format propriétaire .doc pour le dépôt des textes. Ce choix a été guidé par plusieurs constats. D'abord, il s'agit du format le plus répandu dans la communauté visée - pour des raisons que l'on peut déplorer (méconnaissance d'autres possibilités, politique d'achat des institutions, etc.) - et de fait accessible au plus grand nombre d'utilisateurs. Qu'un colloque impose ce format ne peut que contribuer à l'hégémonie actuelle de cet environnement logiciel, et il est envisageable que d'autres choix soient faits à l'avenir. Dans le cas du CMLF'08, le calendrier a joué un rôle important : la plateforme a été mise en place début décembre, puis ouverte à la mi-décembre, pour une date limite de dépôt fixée au 14 janvier. Il fallait donc prendre une décision rapide et lisible. D'autre part, il est apparu nécessaire au comité d'organisation de permettre une relecture des textes eux-mêmes, via des annotations dans le corps de l'article : ce type de relecture n'était pas possible aisément sur des .pdf, par exemple. Il l'était certes sur des documents produits sous Open Office, mais il fallait prévoir ensuite un moyen de les convertir pour que les relecteurs non équipés de ces logiciels puissent les ouvrir. La compatibilité Mac/PC a déjà posé beaucoup de problèmes - sans compter l'accès restreint de Safari, navigateur par défaut du système Mac, à l'interface de dépôt.

Ainsi, pour les prochaines éditions du CMLF, sont à imaginer des solutions techniques fiables et partageables, de sorte que les environnements de travail propres à chacun, pour des raisons pratiques comme pour des raisons idéologiques, soient respectés.

\section{La mise en place des relectures}

La mise en place des relectures a posé des difficultés de divers ordres : la constitution des comités s'est heurtée tout d'abord à des problèmes de calendrier; tous les relecteurs n'ont pas forcément pu être 
contactés à temps, d'autres n'ont pas rendu leurs rapports dans les délais impartis, chacune de ces raisons créant à chaque fois un décalage de la date de notification aux auteurs (laquelle supposait que les comités thématiques disposent de tous les rapports pour pouvoir établir la liste finale des communications retenues).

Les relecteurs devaient, comme les auteurs, se connecter à l'interface de dépôt, qui leur fournissait un login et un mot de passe. Ils disposaient alors d'une page personnelle, laquelle leur donnait accès aux textes qui leur avaient été attribués pour relecture, avec pour chacun : i) un résumé ii) une version de l'article long en .doc, destinée à être annotée puis redéposée sur le site pour que l'auteur y ait accès iii) une version en .pdf pour impression iv) une grille d'évaluation, dont les détails sont donnés infra.

Quelques relecteurs ont abandonné, arguant notamment de difficultés techniques liées au dépôt en ligne des rapports. Dans l'ensemble, très peu de rapports ont dû être déposés par le comité technique (une demi-douzaine de relecteurs concernés sur 167 au total). Les présidents et coordinateurs de thématique ont dans ce cadre constitué un relais efficace, certains ayant pris en charge le dépôt des rapports de leur thématique lorsque cela s'est avéré nécessaire.

\subsubsection{Consignes aux relecteurs}

Les relecteurs ont reçu les instructions suivantes :

«Le CMLF repose sur l'anonymat des propositions mais aussi celui des relectures. Quand vous relisez une proposition, vous avez accès à la fois à une version PDF et à une version .doc (lisible avec Microsoft Word ou OpenOffice Writer). Pour que les propositions retenues soient d'une qualité optimale, nous vous conseillons de récupérer la version .doc et de la reprendre en mode révision avec l'un des deux traitements de texte mentionnés supra. Vous pourrez ainsi directement signaler des scories (langue, présentation) et intégrer aux endroits pertinents vos remarques de fond ou de détail. Le travail de relecture en sera facilité. Et il sera plus utile pour l'auteur. Vous veillerez à faire que ces révisions soient elles aussi anonymes [En effet, par défaut, en mode révision, le nom de l'auteur d'un amendement est indiqué (en utilisant les paramètres de connexion à la machine)]. Un mode d'emploi en ligne [l'URL était fournie] vous indique comment y parvenir. Lorsque la relecture conduit à une proposition de rejet, les annotations peuvent être plus limitées, mais les arguments pour le rejet doivent être explicites et détaillés. Par contre, pour une proposition dont le relecteur souhaite l'acceptation, les commentaires seront aussi précis que possible pour permettre une version finale de qualité. On n'oubliera pas en effet qu'il n'y aura pas de relecture de cette dernière version, en dehors de sa conformité à la feuille de style. Pour les commentaires, on utilisera le mode Révisions pour « incruster » les remarques de forme et de fond directement dans la contribution : cela facilite à la fois le travail du relecteur et celui de l'auteur. On veillera à ce que les révisions restent anonymes en suivant le mode d'emploi qui sera fourni. »

\subsection{La prévention des conflits d'intérêt}

L'anonymat du côté relecture signifie que ni les présidents, ni les coordinateurs, ni les membres de comités thématiques, ni les autres relecteurs ne connaissent les noms des auteurs des propositions. Seuls les présidents du congrès, pour pouvoir prévenir les conflits d'intérêt, savent qui a écrit quoi.

L'examen des propositions a permis en effet aux présidents du congrès d'isoler celles dont les auteurs étaient relecteurs ou membres de comités thématiques. Ces propositions n'ont pas été affectées à des relecteurs par le(s) comité(s) thématique(s) pertinent(s), mais directement par les présidents du congrès. Ces derniers ont augmenté le nombre de relecteurs sollicités : 3 et non 2, comme pour les autres propositions. Ils ont choisi parfois des relecteurs d'autres thématiques mais néanmoins proches des sujets des propositions qui leur étaient soumises. Les relectures ont ensuite été transmises pour décision au(x) comité(s) thématique(s) pertinent(s).

La thématique ayant donné lieu au plus grand nombre de propositions de la part du comité thématique est la syntaxe (6 propositions) ; viennent ensuite la psycholinguistique (4 propositions), la sémantique et la 
morphologie ( 3 propositions), discours et pragmatique ( 2 propositions) quand les autres thématiques n'ont donné lieu qu'à une proposition à conflit d'intérêt potentiel (diachronie, didactique, linguistique textuelle, sociolinguistique). Ces propositions sont le plus souvent le fait d'un seul auteur, avec une exception notable en morphologie, avec 8 coauteurs pour une des propositions concernées.

La prise de décision (acceptation/rejet) par les comités thématiques s'est également faite sans que ces comités connaissent l'identité des auteurs. C'est en effet après cette étape que l"anonymat a été levé.

\subsubsection{Les propositions multi-thématiques}

Le formulaire de dépôt d'une proposition de communication ne permettait de choisir qu'une seule thématique. Par contre, l'auteur pouvait indiquer dans un message qu'à son sens, sa proposition relevait également d'une ou de plusieurs autre(s) thématique(s).

De telles communications ont alors été soumises aux différents comités thématiques concernés, en prévenant chacun qu'il n'avait pas à trouver deux relecteurs pour la proposition, mais un seul puisque la proposition était aussi relue ailleurs.

De manière plus rare, la relecture dans une thématique a conduit un relecteur à proposer une relecture également dans une autre thématique. Dans tous les cas, le comité thématique d'inscription a été destinataire de l'ensemble des relectures et a pris sa décision en conséquence.

Au total, ce sont 35 propositions qui ont été évaluées dans plusieurs thématiques (plus de 13\%).

\subsubsection{Les propositions acceptées}

Les articles d'ouverture (celui des présidents du congrès, le présent article, l'article d'Anne Coste sur le rôle de Ferdinand Brunot dans la création de la Cité universitaire), les conférences plénières transversales ainsi que les points de vue et autres interventions en table ronde n'ont pas donné lieu à évaluation (sauf vérification de la conformité formelle à la maquette des actes). Ce sont donc dans tous les cas des « conférences invitées », relevant de la responsabilité du comité d'organisation dans son ensemble pour les deux premières catégories, de celles de chaque comité thématique pour la dernière. Ce qui suit concerne donc uniquement les propositions qui ont été déposées en ligne en janvier 2008.

Sur une vingtaine de propositions dont l'auteur était relecteur ou membre d'un comité thématique, trois ont été refusées. Le nombre aurait-il été le même si d'une part les propositions n'avaient pas été anonymes et si d'autre part la présidence du congrès n'avait pas affecté elle-même des relecteurs à ces propositions ?

Les retraits après acceptation ont été peu nombreux (moins de cinq) : la raison invoquée par les auteurs concernés est un problème de calendrier (impossibilité de compléter l'article, suivant les remarques des relecteurs, dans les délais impartis, mais également impossibilité d'assister au colloque à la période prévue, pour des raisons personnelles ou professionnelles).

\section{4 Évaluation de l'évaluation}

\subsection{La grille de lecture « interne »}

Les relecteurs avaient à remplir une grille de relecture mise au point par le comité de programme et visant à homogénéiser cette phase.

\subsubsection{Objet ou phénomène linguistiques traités}

Adéquation avec le thème général du colloque («Linguistique française ») :

Adéquation avec la thématique dans laquelle la soumission a été proposée - 
Si mauvaise, thématique(s) plus appropriée(s) :

Clarté d'exposition : (note de 1 à 5 et commentaire éventuel)

\subsubsection{Qualité de rédaction : (note de 1 à 5 et commentaire éventuel)Analyse ou démarche proposées}

Pertinence/importance pour la discipline de l'objet ou du phénomène traités : (note de 1 à 5 et commentaire éventuel)

Maîtrise par l'auteur du sujet traité : (note de 1 à 5 et commentaire éventuel)

Nouveauté du propos : (note de 1 à 5 et commentaire éventuel)

Cadre théorique, selon la pertinence par rapport au sujet traité :

- Cadre explicite / implicite / pas de cadre repérable / non pertinent

- Si cadre, précisez lequel :

Données empiriques, selon la pertinence par rapport au sujet traité :

- $\quad$ avec / sans / non pertinent

- Si avec : nouvelles / connues

\subsubsection{Respect des consignes de présentation}

(section à remplir uniquement si vous acceptez la communication)

1. la feuille de style est-elle bien attachée au document Word? (oui / non)

2. les notes sont-elles effectivement en fin de texte après les références et non en bas de page ? (oui / non)3. les tableaux sont-ils coupés ? (oui / non)4. les images et graphiques sont-ils lisibles ? (oui / non)5. y a-t-il des polices manquantes (elles se signalent par des fragments en symboles exotiques ou en caractères empruntés à d'autres langues)? (oui / non)

6. y a-t-il des polices ou des tailles de police discordantes par rapport à l'ensemble du texte ? (oui / non)

\subsubsection{7. y a-t-il des espaces verticales en trop ? (oui / non)Remarques/suggestions éventuelles réservées au comité scientifique}

\subsection{Indications d'ensemble sur les relectures effectuées}

Dans les tableaux qui suivent, les nombres et pourcentages fournis le sont avant tout à titre indicatif, dans la mesure où les cellules comportent souvent des effectifs restreints. Ils montrent toutefois l'intérêt de disposer d'une chaîne de production de colloques qui restitue des informations quantitatives et qualitatives détaillées et qui facilite l'analyse précise des facteurs à l'oeuvre dans la mise en place d'un colloque.

\subsubsection{Les acceptations}

261 propositions ont été reçues au total. Le taux d'acceptation/rejet est le suivant : 


\begin{tabular}{|l|r|l|}
\hline Statut & nbre & $\%$ \\
\hline accepté & 156 & 59.77 \\
\hline refusé & 105 & 40.23 \\
\hline
\end{tabular}

Le tableau suivant détaille le taux d'acceptation par thématique (classement par taux d'acceptation croissant).

\begin{tabular}{|l|r|r|r|}
\hline Thématique & propositions & acceptées & \% acceptation \\
\hline Lexique(s) & 26 & 9 & 34.62 \\
\hline Phonétique, phonologie & 10 & 5 & 50.00 \\
\hline Sociolinguistique & 15 & 8 & 53.33 \\
\hline Discours & 27 & 15 & 55.56 \\
\hline Sémantique & 41 & 23 & 56.10 \\
\hline Psycholinguistique & 22 & 13 & 59.09 \\
\hline Diachronie & 24 & 15 & 62.50 \\
\hline Didactique & 24 & 15 & 62.50 \\
\hline Texte et écrit & 37 & 26 & 70.27 \\
\hline Histoire, épistémologie & 14 & 10 & 71.43 \\
\hline Morphologie & 7 & 5 & 71.43 \\
\hline Syntaxe & 28 & 22 & 78.57 \\
\hline
\end{tabular}

Une thématique a un taux d'acceptation nettement plus faible que le taux moyen: Lexique(s). La thématique Syntaxe a au contraire un taux d'acceptation beaucoup plus important que le taux moyen.

\subsubsection{Origine géographique}

L'objectif des organisateurs était d'aboutir à un congrès international. Dans les propositions, celles venant de France représentent près de $70 \%$, et celles du reste de l'Europe $15 \%$, soit $15 \%$ pour le reste du monde (3ème colonne). Les proportions ne changent pas notablement sur ces deux points entre les propositions et les communications acceptées (5ème colonne).

Le taux d'acceptation par zone, sur lequel est trié le tableau ci-dessous, varie par contre. Il équivaut au taux moyen pour la France et le reste de l'Europe. Il est très fortement plus faible pour les propositions venant d'Asie, d'Afrique du Nord et du Moyen-Orient, et légèrement plus fort pour les propositions d'Amérique du Nord.

\begin{tabular}{|l|r|r|r|r|r|}
\hline Zone & propositions & $\%$ & acceptées & $\%$ & $\%$ acceptation \\
\hline Amérique S & 4 & 1,53 & 0 & 0 & 0 \\
\hline autre & 1 & 0,38 & 0 & 0 & 0 \\
\hline Asie & 6 & 2,3 & 2 & 1,28 & 33,33 \\
\hline Afr, Moy, Orient & 11 & 4,21 & 4 & 2,56 & 36,36 \\
\hline reste Europe & 39 & 14,94 & 24 & 15,38 & 61,54 \\
\hline France & 181 & 69,35 & 112 & 71,79 & 61,88 \\
\hline Amérique N & 19 & 7,28 & 14 & 8,97 & 73,68 \\
\hline
\end{tabular}

Le tableau suivant détaille par thématique le nombre et la proportion venant de France de propositions et de communications acceptées. Il est classé par ordre croissant de propositions acceptées venant de France. Si l'on ne considère que les communications effectivement acceptées, trois thématiques se détachent par un plus grand nombre de propositions venant du reste de l'Europe ou du monde : Diachronie, Discours et Sociolinguistique, tandis que d'autres au contraire ont un taux d'acceptation de propositions françaises 
notablement plus fort que la moyenne: Syntaxe, Texte et écrit, Didactique, Histoire, épistémologie, Morphologie.

\begin{tabular}{|l|r|r|r|r|r|r|}
\hline Thématique & propositions & France & \% France & acceptées & France & \% France \\
\hline Sociolinguistique & 15 & 9 & 60 & 8 & 4 & 50 \\
\hline Discours & 27 & 14 & 51,85 & 15 & 8 & 53,33 \\
\hline Diachronie & 24 & 11 & 45,83 & 15 & 8 & 53,33 \\
\hline Phonétique, phonologie & 10 & 8 & 80 & 5 & 3 & 60 \\
\hline Lexique(s) & 26 & 17 & 65,38 & 9 & 6 & 66,67 \\
\hline Psycholinguistique & 22 & 16 & 72,73 & 13 & 9 & 69,23 \\
\hline Sémantique & 41 & 27 & 65,85 & 23 & 16 & 69,57 \\
\hline Syntaxe & 28 & 23 & 82,14 & 22 & 18 & 81,82 \\
\hline Texte et écrit & 37 & 29 & 78,38 & 26 & 22 & 84,62 \\
\hline Didactique & 24 & 20 & 83,33 & 15 & 13 & 86,67 \\
\hline Histoire, épistémologie & 14 & 12 & 85,71 & 10 & 9 & 90 \\
\hline Morphologie & 7 & 7 & 100 & 5 & 5 & 100 \\
\hline
\end{tabular}

\subsubsection{Nombre d'auteurs et " générations »}

Les propositions à un seul auteur dominent très largement.

\begin{tabular}{|r|r|r|}
\hline Auteur(s) & propositions & acceptées \\
\hline 1 & 198 & 110 \\
\hline 2 & 32 & 23 \\
\hline 3 & 16 & 12 \\
\hline 4 & 9 & 7 \\
\hline 5 & 1 & 1 \\
\hline 6 & 3 & 1 \\
\hline 7 & 1 & 1 \\
\hline 9 & 1 & 1 \\
\hline
\end{tabular}

Les doctorants ou post-doctorants occupent une place non négligeable parmi les auteurs de propositions et même au sein des communications acceptées.

\begin{tabular}{|r|r|r|r|r|r|}
\hline Propositions & dont néo-doct. & \% néo-doct. & acceptées & dont néo-doct. & \% néo-doct. \\
\hline 261 & 107 & 41.00 & 156 & 58 & 37.18 \\
\hline
\end{tabular}

\subsubsection{Relecteurs et relectures}

Les 167 relecteurs ont effectué 568 relectures (un peu plus de trois en moyenne). Ils avaient la possibilité de reprendre, en mode révision, le texte d'une proposition pour le « farcir» de commentaires précis, situés exactement à l'endroit requis. 73 relecteurs (43.71\%) ont fait de telles modifications, mais pas forcément sur toutes les propositions dont ils avaient la charge. En effet, au total, c'est un tiers des relectures qui ont été accompagnées de révisions directement sur la proposition. 


\begin{tabular}{|r|r|r|r|r|r|r|r|}
\hline relecteurs & modifiant & $\%$ modifiant & relectures & nb. moy. relectures & doc. joints & \% joints \\
\hline 167 & 73 & 43.71 & 568 & 3.4 & 170 & 29.93 \\
\hline
\end{tabular}

L'examen par thématique permet de préciser ces premières tendances. Le nombre moyen de relectures, sur lequel est classé le premier tableau ci-dessous, varie fortement, avec des pressions plus fortes pour Didactique et surtout Sociolinguistique. Il correspond peut-être également à de modes d'organisation différents des comités thématiques: certains se sont adjoints, comme ils en avaient la possibilité, beaucoup de relecteurs (Discours), tandis que d'autres (Sociolinguistique) ont reposé sur un nombre de relecteurs inférieur au nombre de membres du comité thématique (6 personnes). Les thématiques qui ont le plus «farci » les propositions reçues (deuxème tableau infra, classé sur le pourcentage de relecteurs ayant modifié des propositions) sont, par proportion croissante : Histoire, épistémologie, Discours, Texte et écrit et Morphologie. Celles qui ont moins fait appel à ce mode de relecture sont Psycholinguistique, Didactique et Sociolinguistique. Le troisième tableau ci-dessous est organisé par ordre croissant du pourcentage de documents joints aux relectures: faible en Sociolinguistique, il est important pour Discours et Morphologie.

\begin{tabular}{|l|r|r|r|}
\hline Thématique & relecteurs & relectures & nb, moy, relectures \\
\hline Discours & 34 & 50 & 1,5 \\
\hline Morphologie & 10 & 16 & 1,6 \\
\hline Phonétique, phonologie & 10 & 18 & 1,8 \\
\hline Histoire, épistémologie & 12 & 32 & 2,7 \\
\hline Diachronie & 15 & 48 & 3,2 \\
\hline Syntaxe & 15 & 50 & 3,3 \\
\hline Texte et écrit & 14 & 48 & 3,4 \\
\hline Psycholinguistique & 12 & 47 & 3,9 \\
\hline Lexique(s) & 16 & 77 & 4,8 \\
\hline Sémantique & 17 & 89 & 5,2 \\
\hline Didactique & 8 & 48 & 6 \\
\hline Sociolinguistique & 4 & 45 & 11,3 \\
\hline
\end{tabular}

\begin{tabular}{|l|r|r|r|r|}
\hline Thématique & relecteurs & modifiant & \% modifiant & nb, moy, relectures \\
\hline Psycholinguistique & 12 & 3 & 25 & 3,9 \\
\hline Sociolinguistique & 4 & 1 & 25 & 11,3 \\
\hline Didactique & 8 & 2 & 25 & 6 \\
\hline Syntaxe & 15 & 4 & 26,67 & 3,3 \\
\hline Phonétique, phonologie & 10 & 3 & 30 & 1,8 \\
\hline Lexique(s) & 16 & 6 & 37,5 & 4,8 \\
\hline Diachronie & 15 & 7 & 46,67 & 3,2 \\
\hline Sémantique & 17 & 8 & 47,06 & 5,2 \\
\hline Histoire, épistémologie & 12 & 6 & 50 & 2,7 \\
\hline Discours & 34 & 19 & 55,88 & 1,5 \\
\hline Texte et écrit & 14 & 8 & 57,14 & 3,4 \\
\hline Morphologie & 10 & 6 & 60 & 1,6 \\
\hline
\end{tabular}




\begin{tabular}{|l|r|r|r|r|}
\hline Thématique & relecteurs & $n b$, moy, relectures & doc, joints & $\%$ joints \\
\hline Sociolinguistique & 4 & 11,3 & 2 & 4,44 \\
\hline Psycholinguistique & 12 & 3,9 & 6 & 12,77 \\
\hline Didactique & 8 & 6 & 10 & 20,83 \\
\hline Syntaxe & 15 & 3,3 & 12 & 24 \\
\hline Phonétique, phonologie & 10 & 1,8 & 5 & 27,78 \\
\hline Sémantique & 17 & 5,2 & 25 & 28,09 \\
\hline Lexique(s) & 16 & 4,8 & 23 & 29,87 \\
\hline Histoire, épistémologie & 12 & 2,7 & 12 & 37,5 \\
\hline Texte et écrit & 14 & 3,4 & 19 & 39,58 \\
\hline Diachronie & 15 & 3,2 & 19 & 39,58 \\
\hline Discours & 34 & 1,5 & 27 & 54 \\
\hline Morphologie & 10 & 1,6 & 10 & 62,5 \\
\hline
\end{tabular}

On peut examiner la taille des relectures « externes », c'est-à-dire la partie de la relecture qui est destinée à l'auteur. Elle peut être très longue (1 500 mots, soit environ 3 pages), mais est en moyenne courte (106 mots soit 3 phrases environ), et $8.66 \%$ des relectures sont vides, c'est-à-dire ne comportent pas du tout de remarques pour l'auteur.

\begin{tabular}{|r|r|r|r|r|}
\hline Relectures externes & max. mots & nbre moy. mots & vides & $\%$ vides \\
\hline 566 & $1,500.00$ & 106.81 & 49 & 8.66 \\
\hline
\end{tabular}

Les pratiques de relectures ne sont pas homogènes. On note en effet des variations importantes dans le nombre moyen de mots par relecture externe (un rapport de un: Phonétique, phonologie à 5 : Morphologie) et dans le nombre de relectures «vides» (de 0 à $36.17 \%$ ). Le premier tableau ci-dessous est trié par ordre croissant du nombre moyen de mots de la relecture. Le deuxième tableau l'est par pourcentage décroissant de relectures externes vides.

\begin{tabular}{|l|r|r|r|}
\hline Thématique & relectures externes & max, mots & nbre moy, mots \\
\hline Phonétique, phonologie & 18 & 246,75 & 44,38 \\
\hline Psycholinguistique & 47 & 191,25 & 57,83 \\
\hline Sociolinguistique & 45 & 380,25 & 72,13 \\
\hline Texte et écrit & 48 & 332,13 & 75,4 \\
\hline Didactique & 48 & 259,88 & 80,35 \\
\hline Diachronie & 48 & 736 & 92,39 \\
\hline Syntaxe & 50 & $1,083,38$ & 102,3 \\
\hline Histoire, épistémologie & 32 & 647,38 & 110,65 \\
\hline Lexique(s) & 77 & 692,75 & 113,78 \\
\hline Sémantique & 87 & 681,13 & 121,3 \\
\hline Discours & 50 & $1,500,00$ & 198,64 \\
\hline Morphologie & 16 & $1,239,63$ & 242,33 \\
\hline
\end{tabular}




\begin{tabular}{|l|r|r|r|}
\hline Thématique & relectures externes & vides & \% vides \\
\hline Psycholinguistique & 47 & 17 & 36,17 \\
\hline Morphologie & 16 & 3 & 18,75 \\
\hline Syntaxe & 50 & 9 & 18 \\
\hline Didactique & 48 & 7 & 14,58 \\
\hline Histoire, épistémologie & 32 & 4 & 12,5 \\
\hline Diachronie & 48 & 3 & 6,25 \\
\hline Phonétique, phonologie & 18 & 1 & 5,56 \\
\hline Lexique(s) & 77 & 3 & 3,9 \\
\hline Discours & 50 & 1 & 2 \\
\hline Sémantique & 87 & 1 & 1,15 \\
\hline Texte et écrit & 48 & 0 & 0 \\
\hline Sociolinguistique & 45 & 0 & 0 \\
\hline
\end{tabular}

\subsection{Les avis aux auteurs}

Les avis des relecteurs ne facilitent pas toujours la compréhension par l'auteur de la décision d'acceptation ou surtout de refus par le comité thématique. Leur taille est très variable (cf. supra). Ils ne donnent pas forcément un avis suffisamment précis pour étayer la décision proposée et matérialisée par un choix dans une liste à choix. Le comité thématique s'est appuyé dans son choix final sur la connaissance de la partie « interne» de l'évaluation, mais ne disposait pas toujours d'éléments concordants dans la partie « externe » permettant à l'auteur de saisir le bien-fondé de la décision qui lui était notifiée. D'où un certain nombre de réactions légitimes et auxquelles le comité thématique n'était pas en mesure de fournir des réponses adéquates.

A contrario, plus rarement, mais on espère qu'une prochaine édition sera l'occasion d'un accroissement significatif, nous avons rencontrés des relus heureux, y compris une personne dont la proposition a été refusée. Ces auteurs étaient comblés de bénéficier de relectures minutieuses, précises, les aidant à progresser.

La composition des comités thématiques visait à exclure la prédominance de telle ou telle position théorique dans le champ concerné. Les divergences d'appartenance théorique ne semblent pas avoir joué un rôle important dans les refus : peu de réclamations relèvent de cette catégorie.

\section{Conclusions et perspectives}

\section{1 Écarts entre les usages et les techniques disponibles}

À l'ère du Web collaboratif (dit 2.0), celui de Wikipedia, du partage et de l'annotation collaborative, folksonomique, d'images (Flickr - http://www.flickr.com/) ou de signets (del.icio.us - http://del.icio.us/), certains thuriféraires de la modernité triomphante font l'opposition entre les Digital Natives et Digital Immigrants, les « indigènes (autochtones ?) du numérique » et les « immigrants du numérique », ceux qui sont tombés tout petits dans le numérique, et ceux pour qui c'est un monde toujours nouveau, parfois saumâtre, inquiétant bien souvent.

Dans l'utilisation concrète de la plateforme, environ un quart des relecteurs ont eu besoin, à un titre ou à un autre, d'aide technique, pour la moitié environ des auteurs. L'interaction par courriel avec la personne en charge de l'aide a d'ailleurs donné lieu à des formes d' « incivilité numérique » : tout se passe comme si l'écrit immédiat du courriel rendait aisés, sinon licites, des débordements par rapport à la correspondance écrite. Cet agacement des auteurs, voire ce désarroi, tendrait à prouver que les linguistes, de France, de Navarre et d'ailleurs, sont représentatifs des usages grand public de l'informatique et donc se situent plutôt du côté des «immigrants » du numérique. Il n'est d'ailleurs même pas sûr que les plus jeunes dans les 
auteurs ayant proposé des communications au CMLF'08 ressortissent vraiment aux « indigènes » du numérique. Au moins en France, leur formation proprement universitaire ne les prépare pas forcément à une compréhension et une manipulation aisée d'environnements de travail comme la plateforme de dépôt. L'instauration d'un «permis de conduire informatique » obligatoire en université, le C2I (Certification Informatique et Internet - http://www2.c2i.education.fr/), est d'ailleurs relativement récente (les textes réglementaires sont de 2002) et les ambitions dudit permis relativement modestes.

Plutôt que d'une distinction statique, suspecte de jeunisme et de technophilie débridée, sans doute faudrait-il parler d'une «barrière mobile », qui se déplace d'un individu à l'autre, au sein d'une même « génération » et qui bouge pour un individu donné. On peut progresser et tout aussi bien régresser, ne serait-ce que parce que les environnements évoluent sans cesse : nous sommes tous, ou nous serons tous un jour ou l'autre, des «immigrants» du numérique. En tout cas, un préalable, éviter les doubles nécessaires de l'enthousiasme technophile : le rejet en bloc, la distinction entre le haut (les opérations de pensée) et le bas (les dispositifs matériels de la pensée). Pour reprendre Montaigne (Les Essais, Livre III, ch. XI) : « ... tous les abus du monde s'engendrent, de ce qu'on nous apprend à craindre de faire profession de nostre ignorance... » Il y a certainement, dans la lignée du livre de Gilbert Simondon (Du mode d'existence des objets techniques, Aubier, 1958) ou du premier volet de Lieux de savoir dirigé par Christian Jacob (Albin Michel, 2007), à penser, au sens le plus strict et le plus fort, les places possibles d'environnements de travail comme une plateforme de gestion de colloques en ligne. Il ne s'agit d'ailleurs pas plus de vivre ces conditions d'interaction comme des « destins », mais comme des objets techniques dont les usages sont à réfléchir, pour des évolutions conjointes des usages et des dispositifs techniques.

\subsection{Pouvoir évaluer l'évaluation}

En traitement automatique des langues et en ingénierie linguistique comme en recherche et extraction d'information, depuis plus d'une dizaine d'années, les approches proposées, qu'il s'agisse de recherches expérimentales ou de modules plus applicatifs, s'inscrivent dans une démarche d'évaluation. Il s'agit d'étalonner les performances observées par rapport à une « pierre de touche » déterminée à l'avance. Par exemple, on mettra au point un corpus de référence de textes dont l'étiquetage morpho-syntaxique a été vérifié manuellement et on examinera les performances d'étiqueteurs automatiques par comparaison avec ce corpus de référence. À ce niveau de performance globale, on cherche également à adjoindre d'autres indications plus fines, sur le comportement pas à pas des logiciels en cause. Il s'agit en quelque sorte d'« ouvrir le capot» et d'identifier les performances des composants du système utilisé. L'intérêt de disposer d'une plateforme logicielle comme celle d'Intellagence est du même ordre. Une telle plateforme ne rend pas l'évaluation plus objective: il s'agit toujours de jugements humains, teintés parfois de préjugés théoriques ou effectués trop rapidement. L'évaluation devient par contre plus objectivable. On peut l'examiner pas à pas, déterminer les points de faiblesse. La proportion de communications acceptées par rapport au nombre de propositions constitue d'ailleurs un indicateur sans vraie substance : elle varie trop selon la «pression », le nombre de publiants potentiels, du domaine concerné. Il faut s'interroger surtout sur la qualité des relectures. Si certains auteurs ont fait connaître leur satisfaction d'avoir été vraiment relus « en profondeur », d'autres, à juste titre, ont eu le sentiment d'une acceptation ou d'un refus peu motivé(e). La culture de la relecture est inégalement répartie au sein des relecteurs qui ont contribué au CMLF, peut-être parce que, dans l'immédiat, la relecture est un versant peu valorisé voire « valorisable » d'une carrière.

\section{3 Écrire, parler, écouter, questionner : vers de nouveaux réglages}

L'inquiétude de certains auteurs ou membres de comités thématiques est qu'à disposer ainsi des articles dès l'ouverture du congrès s'évide ce qui fait l'intérêt de telles rencontres : l'écoute du neuf, la réaction à la parole vive, l'ajustement au public et à ses réactions. Le risque est réel de la simple accumulation des publications, à l'heure justement où seul semble compter le compte (l'obsession bibliométrique).

Dans le même temps, la connaissance fine des avancées en sciences du langage n'est pour le moment guère facilitée par la multiplicité des «À paraître », par le temps qui sépare les colloques de leur 
publication (quand publication il peut y avoir !) et par la difficulté à avoir accès aux articles. La complémentarité du numérique par rapport aux autres supports doit être mise maximalement à contribution pour améliorer la construction des connaissances via leur échange et leur critique. Les auteurs sont d'ailleurs explicitement invités à reprendre et à faire évoluer la contribution qui figure dans les actes du CMLF'08.

Le fait de disposer à l'avance des communications par ailleurs présente l'intérêt de pouvoir, pour une thématique donnée, essayer de faire le bilan de ce qu'elles révèlent de l'état de cette thématique. C'est ce qui a été demandé aux comités thématiques pour les tables rondes conclusives. On peut imaginer aussi la possibilité de «discutants», c'est-à-dire de personnes qui ont lu à l'avance une communication et qui, parallèlement aux questions spontanées, proposent une mise en perspective et des liens avec d'autres communications de la même session.

\subsection{Quel rapport qualité/prix ?}

L'utilisation de la plateforme a connu des à coups techniques. A choqué l'envoi initial de messages en anglais, lié au fait que la plateforme sert plus souvent pour des disciplines où l'anglais est la langue de communication. La gestion de plusieurs comités thématiques, fonctionnant comme autant de comités de programme séparés, et distincts en ce sens de simples sessions sur un sujet déterminées par un comité de programme unique, n'est pas immédiate dans l'état actuel de la plateforme. Il en est résulté d'une part des demandes relativement spécifiques et fréquentes à la personne support du CMLF à Intellagence et d'autre part, sans doute, des pertes de temps liées à la coordination des opérations entre les thématiques. Découpler partiellement les thématiques aurait sans doute permis, au total, de gagner du temps.

Au delà des dysfonctionnements techniques, il $\mathrm{y} \mathrm{a}$ eu, pour une proportion non négligeable d'auteurs mais aussi, moins fortement, de relecteurs ou de membres de comités thématiques, la difficulté à comprendre la nécessité et les impératifs d'une chaîne de traitement des propositions reposant sur un découpage relativement strict des tâches. La tentation était grande de s'adresser à la personne qu'on connaissait plutôt qu'à la personne en charge. D'où du temps supplémentaire à re-diriger les informations pertinentes à la personne concernée. Sans doute faudrait-il que les messages automatiques envoyés par la plateforme (accusé de réception d'une proposition, assignation de relectures, indication d'acceptation ou de rejet) soient signés par la ou les personnes pertinentes. Sans doute aussi faut-il indiquer au destinataire quand il a affaire à un message "pré-fabriqué », envoyé automatiquement par la plateforme après paramétrage éventuel, et quand il s'agit d'un message « humain ».

Un bilan «comptable» du recours à la plateforme de colloques en ligne d'Intellagence est difficile. Se trouvent en effet concentrées sur un temps très restreint $(7$ mois) des opérations qui habituellement s'écoulent sur plusieurs années. Ordinairement, appel à communications, choix des propositions, colloque à proprement parler constituent la première phase. Une deuxième phase intervient notablement plus tard, une fois obtenus les crédits pour la publication ou l'accord d'un éditeur : choix des contributions à intégrer dans la publication, va-et-vient éventuel de relectures et d'améliorations, « peignage » présentationnel des fichiers reçus pour l'envoi à l'impression, impression et diffusion. Les interventions sont multiples, les rôles imprécis (un responsable éditorial peut tout aussi bien régler un problème de feuille de style). L'investissement fait par l'ILF dans une gestion en ligne du colloque et une publication numérique (sur CD-Rom et sur un site) serait à comparer avec un décompte fin des temps et des compétences engagés dans un mode de gestion traditionnel. 